\title{
O tratamento clássico do interferômetro de Mach-Zehnder: uma releitura mais moderna do experimento da fenda dupla na introdução da física quântica (The optical Mach-Zehnder interferometer in classical regime: a more modern version of the double-slit experiment in the introduction of quantum physics)
}

\author{
Trieste Freire Ricci ${ }^{1}$, Fernanda Ostermann e Sandra Denise Prado \\ Instituto de Física, Universidade Federal do Rio Grande do Sul, Porto Alegre, RS, Brasil \\ Recebido em 2/6/2006; Aceito em 21/8/2006
}

\begin{abstract}
Neste trabalho, analisamos detalhadamente o fenômeno ondulatório da luz em um interferômetro de MachZehnder (IMZ) operando em regime clássico. Um dos principais objetivos do trabalho é dar suporte a futuras transposições para o IMZ em regime quântico. O IMZ é um arranjo experimental que apresenta uma releitura, mais moderna em termos tecnológicos, do experimento das duas fendas de Young [1] e tem sido extensivamente explorado em artigos da área de computação quântica e criptografia quântica [2] - áreas que se alicerçam nos fenômenos de superposição e de interferência quânticas - e em artigos de ensino de física para disciplinas introdutórias de física quântica (FQ) - área que propõe naturalmente uma reflexão sobre o problema conceitual da escolha dos caminhos pelo fóton [3-10]. Os resultados analíticos aqui apresentados foram utilizados na elaboração de um software educacional que simula a interferência nos regimes clássico e quântico e que está em plena utilização em cursos introdutórios de FQ no IF-UFRGS.
\end{abstract}

Palavras-chave: óptica ondulatória, interferômetro de Mach-Zehnder, dualidade onda-partícula.

In this paper, the optical Mach-Zehnder Interferometer (MZI) in classical regime is fully analysed. One of our main goals is to supply the basis for future transpositions to the quantum regime. The MZI is a device totally analogous to the double-slit experiment [1] but a more modern version in technological terms. It has been used extensively in papers related to quantum computation and quantum cryptography [2]. Those are fields grounded on quantum superposition and quantum interference phenomena. It has also appeared in papers related to the teaching of introductory quantum physics where the conceptual problem of which path a given photon goes through arises more naturally [3-10]. The analytical results shown here were used in the production of an educational software to simulate the classical and quantum interference phenomena. The software has been widely used in introductory quantum physics courses in IF-UFRGS.

Keywords: optical physics, Mach-Zehnder interferometer, wave-particle duality.

\section{Introdução}

O IMZ é um arranjo relativamente simples de espelhos e semi-espelhos que demonstra claramente o fenômeno da interferência da luz pela divisão de um feixe luminoso. Este é dividido em um primeiro semi-espelho e então recombinado em um segundo semi-espelho (Fig. 1) onde, dependendo da fase relativa adquirida pelos feixes ao longo dos caminhos, exibirá interferência construtiva com eficiência entre 0 e 100\%. Com esse aparato, idealizado independentemente por Ludwig Zehnder (18541949) [11] e Ludwig Mach (1868-1951) [12] em 1891/92, pode-se, por exemplo, monitorar alterações mínimas no índice de refração e na compressibilidade de um fluxo

\footnotetext{
${ }^{1}$ E-mail: ricci@if.ufrgs.br.

de gás, sendo muito utilizado em aerodinâmica. Exceto por citações de aplicações em áreas específicas, o IMZ parece não ser nem mesmo mencionado nos livros de física amplamente adotados no nível de graduação - é indiscutível a preferência pelo arranjo da fenda dupla, tanto em abordagens da óptica ondulatória quanto da física moderna [13-15]. A originalidade deste trabalho consiste em apresentar o tratamento clássico do IMZ de forma a se constituir em material didático de apoio, tanto para o estudante quanto para o professor, em cursos introdutórios de FQ.

A primazia do arranjo da fenda dupla para o caso da luz pode ser facilmente entendida, uma vez que, além de menções históricas que remetem a Young nos 
primórdios de 1800, a geometria da fenda dupla é matematicamente mais acessível aos estudantes no nível das Físicas Gerais do que a de aberturas circulares, que é o caso do IMZ. Na FQ, por outro lado, o arranjo da fenda dupla para elétrons foi consagrado por Feynman em sua série de livros publicada em 1963 [16], tornandose, assim, freqüentemente usada em livros-texto e em discussões em sala de aula - principalmente quando o ponto de partida para a introdução aos conceitos da FQ é a óptica ondulatória [3-10 e 13-15]. Curiosamente, o editorial da revista Physics World de setembro de 2002 chama a atenção para o fato de que o primeiro experimento de interferência de elétrons foi feito quase 10 anos antes da série de aulas de Feynman, nos primórdios de 1950, por Ladislaus Laszlo Marton, nos Estados Unidos e usando um IMZ em vez da fenda dupla [17]. As razões pelas quais a interferometria eletrônica é pouco citada nos livros-texto não-técnicos, assim como questões pertinentes ao seu desenvolvimento tecnológico, estão além do objetivo deste artigo, mas a interferometria atômica tem sido tema de muitos artigos em periódicos importantes [18]. O IMZ, por sua vez, tem sido tema freqüente em artigos nas áreas de computação e criptografia quântica $[1,2]$, sendo, portanto, um tema extremamente atual.

Nosso objetivo neste artigo é mostrar que o IMZ em regime clássico é factível de ser abordado por ocasião do primeiro contato dos estudantes com a óptica ondulatória. Apesar do uso de funções especiais para a descrição dos anéis de interferência, como a função de Airy, mostramos que o IMZ é, do ponto de vista geométrico, totalmente análogo ao arranjo de uma fenda dupla. Se bem explorada tal analogia (explicada na primeira parte da seção 2), permite que o estudante interessado apenas em usar o simulador do IMZ evite a segunda parte dessa seção, onde os cálculos detalhados são apresentados.

Além disso, esse material didático vem fornecer subsídios ao uso do software livre Interferômetro Virtual de Mach-Zehnder [18-20], que é uma bancada virtual disponibilizada em Português, Espanhol e Inglês, operando nos regimes clássico e quântico, dependendo da opção do usuário, e com ampla utilização de polaróides (em ambos os regimes) e de detectores (em regime quântico). Bancadas virtuais que exploram o IMZ em regimes clássico e quântico para o ensino da FQ introdutória são temas relativamente recentes na literatura voltada ao ensino de FQ, como por exemplo, o IMZ de Müller e Wiesner [6, 7]. Desenvolvemos uma versão mais completa do IMZ em relação à do software de Müller e Wiesner, com correções de alguns bugs de programação que havia no software citado e importantes generalizações do arranjo considerado, além de inúmeras possibilidades novas de uso de polaróides e de detectores, tanto em regime clássico quanto em regime quântico. Ressaltamos que o IMZ em regime clássico pode servir como excelente ponto de partida para a discussão da dualidade onda-partícula na FQ, graças ao fato de que perguntas tais como por qual braço rumou o fóton? [3-10] são mais naturalmente provocadas por esse arranjo.

No que segue, na seção 2, analisamos o IMZ com uma fonte polarizada, em regime clássico e com todos os detalhes de cálculo que permitem uma compreensão mais profunda dos resultados observados nas simulações, com e sem a presença de filtros polaróides. Na seção 3, dentre uma gama de possibilidades de utilização do simulador operando no regime clássico, escolhemos algumas situações que reforçam os conceitos acerca do caráter ondulatório da luz e também as condições necessárias para que ocorra o fenômeno da interferência. Finalmente, apresentamos nossas considerações finais na seção 4 .

\section{O IMZ sob o ponto de vista da óptica ondulatória}

Vamos iniciar considerando o IMZ esquematizado na Fig. 1. Sob condições idealizadas, os espelhos $M_{1}$ e $\mathrm{M}_{2}$ são considerados perfeitamente refletores e também se considera que os semi-espelhos $\mathrm{SM}_{1}$ e $\mathrm{SM}_{2}$ refletem e transmitem exatamente $50 \%$ da luz neles incidente. Cada braço do interferômetro tem um mesmo comprimento $L$ (medido entre os pontos centrais de um espelho e de um semi-espelho, ao longo do eixo de simetria central de cada braço); e $D$ é a distância entre o ponto central de $\mathrm{SM}_{2}$ ao centro do anteparo 1 ou do anteparo 2 .

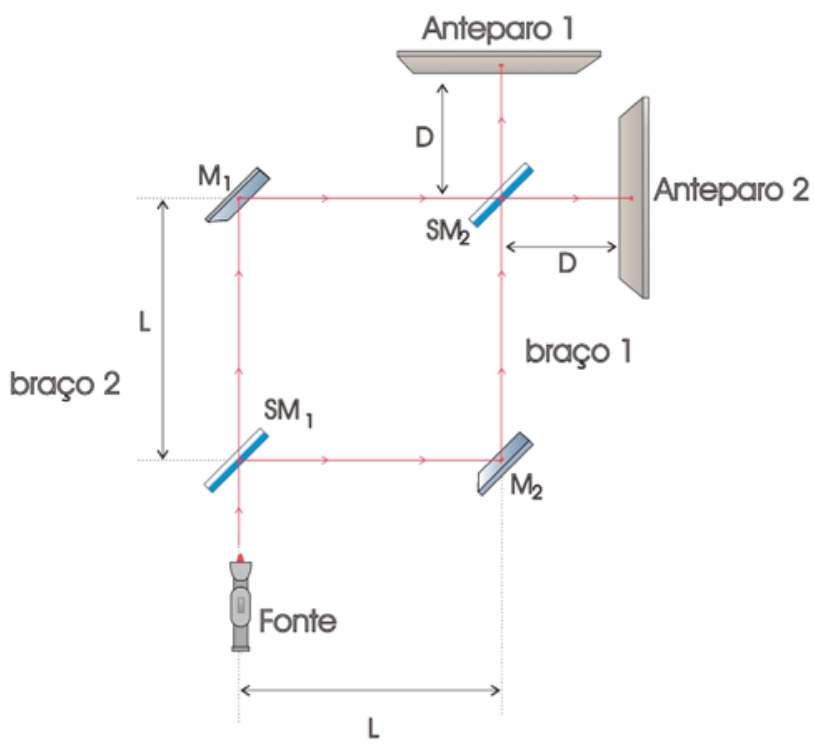

Figura 1 - Esquema de um interferômetro de Mach-Zehnder.

Quanto à fonte, vamos considerar inicialmente que ela seja, simplesmente, uma fonte puntiforme de luz monocromática (comprimento de onda igual a $\lambda$ ) polarizada na direção horizontal (eixo $x$ ) e de intensidade igual a $2 I_{0}$ - de maneira que $I_{0}$ será a intensidade lumi- 
nosa do feixe que entra em cada braço, após o primeiro semi-espelho. Após o segundo semi-espelho, o feixe proveniente de cada braço será dividido novamente em dois feixes, cada qual de intensidade $I_{0} / 2$. Inicialmente, por se tratar de uma fonte puntiforme, não precisaremos nos preocupar com efeitos de difração, como os que ocorrem na saída de uma fonte luminosa real e nãopuntiforme (mais adiante, consideraremos o caso mais realístico em que a fonte não é puntiforme e introduz efeitos de difração nos padrões que se observam nos anteparos).

A análise do funcionamento do IMZ é bastante simplificada quando nos damos conta de que, do ponto de vista dos dois raios luminosos que chegam a um ponto qualquer de um dos anteparos, o interferômetro de IMZ é fisicamente equivalente a um arranjo de fenda dupla em que as "fendas" são duas fontes puntiformes, coerentes, monocromáticas, de mesma freqüência e de intensidades iguais a $I_{0} / 2$. Para o caso mais simples de um raio luminoso emitido pela fonte puntiforme real do IMZ na direção do eixo central do mesmo e que, portanto, atinge o centro do anteparo 1 , é fácil verificar que o interferômetro é fisicamente equivalente a um arranjo de duas fontes puntiformes, coerentes e monocromáticas (comprimento de onda $\lambda$ ), que emitem dois raios luminosos a partir de uma mesma localização, a uma distância $2 L+D$ do anteparo. Neste caso, é fácil se convencer de que esses raios chegam em fase ao ponto central do anteparo. A diferença de fase nula se deve a dois fatores:

(i) não existe diferença de fase devido a uma diferença de caminho entre os raios luminosos que seguiram pelos braços 1 e 2 do IMZ, pois ambos percorreram a distância $r_{o}=2 L+D$;

(ii) cada reflexão da luz em um espelho ou semiespelho introduz uma diferença de fase correspondente a $\lambda / 4$, ou seja, uma diferença de fase igual a $\pi / 2[21]$. Dessa maneira, os raios de luz que seguem pelos dois braços do IMZ e que chegam ao centro do anteparo 1 sofrem, ambos, duas reflexões pelo caminho, de maneira que a diferença de fase final entre eles segue sendo nula.

Por outro lado, para os raios correspondentes e que chegam ao centro do anteparo 2, o IMZ equivale ao mesmo arranjo descrito acima, mas com uma das fontes emitindo luz com diferença de fase inicial de $\pi$ radianos em relação à outra. Essa diferença de fase não nula expressa o fato de que, para chegar ao ponto central do anteparo 2, o raio que vai pelo braço 1 sofre três reflexões, enquanto o que vai pelo braço 2 sofre apenas uma. Isso resulta em uma diferença de fase líquida e adicional de $\pi$ radianos entre os dois raios que chegam ao centro do anteparo 2. (Como para o caso de um ponto central do anteparo 1 , nenhuma diferença de fase é originada por diferença de caminhos, pois os compri- mentos dos caminhos seguidos pelos raios através dos dois braços continuam sendo exatamente iguais.)

Chegamos à conclusão, portanto, de que, no ponto central do anteparo 1, ocorre interferência construtiva entre os dois raios (ponto luminoso no centro do anteparo), enquanto no ponto central do anteparo 2 ocorre interferência destrutiva entre os raios luminosos (ponto não-iluminado no centro do anteparo). Agora prossigamos a análise considerando pontos não-centrais dos dois anteparos. Novamente, usaremos um arranjo de duas fontes puntiformes fisicamente equivalente ao IMZ para este caso. Vamos considerar um raio luminoso emitido pela fonte real segundo um ângulo $\theta$ com o eixo central do IMZ. Depois do raio se dividir em dois, no primeiro semi-espelho, os raios resultantes seguem pelos braços do interferômetro e chegam ao anteparo 1 em um ponto $\mathrm{P}_{1}$ não central, situado a uma distância $\rho$ do centro do mesmo. O leitor pode se convencer disso fazendo um desenho do IMZ que mostre o caminho seguido pelo raio central (chegando ao centro do anteparo) e os caminhos seguidos pelo raio não central que se dividiu e seguiu através dos dois braços (chegando a $\mathrm{P}_{1}$ não central), fazendo uso da lei da reflexão em cada reflexão em espelho ou semi-espelho. Os caminhos percorridos pelos dois raios, $r_{1}$ e $r_{2}$, são diferentes entre si (e ambos maiores do que $r_{o}$ ), resultando em uma diferença de fase entre dois. O arranjo de fenda dupla equivalente está mostrado na Fig. 2 (que não está em escala!). A distância $d$ entre as fontes fictícias $\mathrm{F}_{1}$ e $\mathrm{F}_{2}$ pode ser calculada aproximadamente e de maneira muito simples quando o ponto $\mathrm{P}_{1}$ pertence às primeiras franjas não centrais do padrão de interferência no anteparo, o que corresponde à situação em que o ângulo $\theta$ é muito pequeno. Este é o caso de interesse prático, de fato, uma vez que, se tais ângulos não forem muito pequenos, os raios correspondentes não chegarão ao anteparo, seja por incidirem fora do mesmo ou fora de algum dos espelhos e semi-espelhos do IMZ. Existe uma razão adicional para considerarmos apenas os primeiros máximos não centrais do padrão de interferência: na situação mais realística em que uma fonte luminosa extensa e não puntiforme é usada no IMZ, os efeitos de difração se manifestam através da diminuição progressiva da intensidade máxima de cada máximo lateral de interferência, em relação à intensidade do máximo central. Dessa maneira, na prática, apenas os primeiros máximos não centrais do padrão serão perceptíveis ao olho humano. Sendo assim, os ângulos $\theta_{2}$ e $\theta_{1}$ da Fig. 2, embora diferentes entre si, e também de $\theta$, também são muito pequenos, de modo que o ponto $\mathrm{P}_{1}$ deve estar ligeiramente acima do ponto do anteparo situado diretamente à frente de luz fictícia $\mathrm{F}_{1}$. Ou seja, a distância $\rho$ deste ponto ao centro do anteparo é, com boa aproximação, igual $d / 2$, portanto, $d \cong 2 \rho$. 


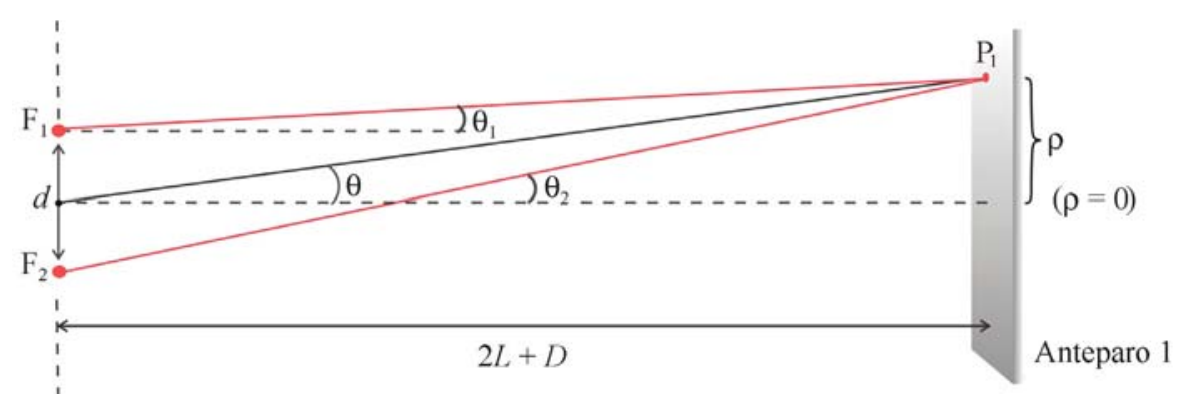

Figura 2 - Arranjo de duas fontes equivalente ao IMZ para o caso de um $\mathrm{P}_{1}$ ponto não central do anteparo 1. A figura não está em escala, uma vez que se considera sejam satisfeitas as condições $r_{o}=2 L+D \gg d$ e $r_{o} \gg \rho$, de maneira que os ângulos assinalados são, em realidade, muito pequenos.

Como antes, as fontes puntiformes fictícias $F_{1}$ e $F_{2}$ são consideradas puntiformes, monocromáticas (e de mesmo comprimento de onda), coerentes entre si e polarizadas na direção horizontal. Mas agora existe uma diferença de fase entre os dois raios que chegam a $\mathrm{P}_{1}$ devido à diferença dos caminhos, $\Delta r=r_{2}-r_{1}$, seguidos pelos raios, pois as fontes fictícias agora não emitem a partir de um mesmo lugar. Para raios luminosos que chegam a um ponto $\mathrm{P}_{2}$ não central do anteparo 2 , o IMZ também equivale a um arranjo de duas fendas como o da Fig. 2, mas onde, além da diferença de fase devido à diferença de caminhos, existe uma diferença de fase adicional de $\pi$ radianos devido ao número diferente de reflexões nos espelhos e semi-espelhos dos braços. A diferença de fase correspondente será dada por

$$
\Delta \phi=k \Delta r=\frac{2 \pi}{\lambda} \Delta r .
$$

As diferenças de fase $\Delta \phi_{1}$ e $\Delta \phi_{2}$ entre os raios luminosos que chegam a um ponto $\mathrm{P}_{1}$ não central do anteparo 1 e a um ponto $\mathrm{P}_{2}$ não central do anteparo 2 , ambos situados a uma mesma distância $\rho$ dos respectivos centros dos anteparos, são dadas, respectivamente, por

$$
\Delta \phi_{1}=\frac{2 \pi}{\lambda} \Delta r \quad \text { e } \quad \Delta \phi_{2}=\Delta \phi_{1}+\pi .
$$

Para a situação discutida antes, em que os ângulos $\theta_{1}, \theta_{2}$ e $\theta$ da Fig. 2 são muito pequenos, a diferença de caminho para os dois raios luminosos é dada aproximadamente por [13]

$$
\Delta r \cong d \operatorname{sen}(\theta) \cong 2 \rho \operatorname{tg}(\theta)=\frac{2 \rho^{2}}{(2 L+D)},
$$

de modo que

$$
\Delta \phi_{1}=\frac{4 \pi}{\lambda(2 L+D)} \rho^{2}=a \rho^{2},
$$

onde $a=4 \pi / \lambda(2 D+L)$ é uma constante.

Assim, em um ponto $\mathrm{P}_{1}$ não central do anteparo 1 , os campos elétricos instantâneos $\mathbf{E}_{1}\left(P_{1}, t\right)$ e $\mathbf{E}_{2}\left(P_{1}, t\right)$ das ondas luminosas que ali chegam, provenientes dos braços 1 e 2 do IMZ, se propagam em direções muito parecidas, dadas respectivamente pelos vetores de onda $\boldsymbol{k}_{1}$ e $\boldsymbol{k}_{2}$. Além disso, esses campos têm praticamente a mesma amplitude $E_{0}$ em $\mathrm{P}_{1}$, pois saíram das duas fontes com a mesma amplitude inicial e percorreram distâncias praticamente iguais até $\mathrm{P}_{1}$, de modo que sofreram praticamente a mesma diminuição (relacionada à diminuição da intensidade luminosa). Finalmente, uma vez que a luz emitida pela fonte tem polarização horizontal (eixo $x$ ), seu campo elétrico tem a direção do vetor unitário $\boldsymbol{e}_{x}$. Portanto, podemos representar matematicamente esses campos elétricos, em um ponto não central do anteparo 1 e em um instante de tempo $t$, pelas expressões

$$
\mathbf{E}_{1}\left(P_{1}, t\right)=\frac{E_{0}}{\sqrt{2}} E_{0} \cos \left(\omega t-\boldsymbol{k}_{1} \cdot \boldsymbol{r}\right) \quad \hat{\boldsymbol{e}}_{x}
$$

e

$$
\mathbf{E}_{2}\left(P_{1}, t\right) \cong \frac{E_{0}}{\sqrt{2}} \cos \left(\omega t-\boldsymbol{k}_{1} \cdot \boldsymbol{r}+\Delta \phi_{1}\right) \hat{\boldsymbol{e}}_{x}
$$

onde $E_{0}$ é a amplitude do campo na entrada de cada braço do interferômetro, de modo que $I_{0} \propto E_{0}^{2} / 2$ [13]. A razão do fator $1 / \sqrt{2}$ nas expressões acima é que elas descrevem os campos após o segundo semi-espelho que divide a intensidade de cada feixe pela metade e, portanto, reduz a amplitude do campo correspondente pelo fator $\sqrt{2}$. O sinal de aproximação usado na expressão para $\mathbf{E}_{2}\left(\mathrm{P}_{1}, t\right)$ se deve ao fato de que efetuamos a aproximação $\boldsymbol{k}_{2} \cong \boldsymbol{k}_{1}$, válida para o caso de interesse em que os ângulos são muito pequenos. Para um ponto $\mathrm{P}_{2}$ não central do anteparo 2 , os correspondentes campos elétricos instantâneos das ondas eletromagnéticas que seguiram pelos braços 1 e 2 são, respectivamente, representados pelas expressões

$$
\mathbf{E}_{1}\left(P_{2}, t\right)=\frac{E_{0}}{\sqrt{2}} \cos \left(\omega t-\boldsymbol{k}_{1} \cdot \boldsymbol{r}\right) \hat{\boldsymbol{e}}_{x}
$$

e 


$$
\begin{aligned}
& \mathbf{E}_{2}\left(P_{2}, t\right) \cong \frac{E_{0}}{\sqrt{2}} \cos \left(\omega t-\boldsymbol{k}_{1} \cdot \boldsymbol{r}+\Delta \phi_{1}+\pi\right) \hat{\boldsymbol{e}}_{x}= \\
& -\frac{E_{0}}{\sqrt{2}} \cos \left(\omega t-\boldsymbol{k}_{1} \cdot \boldsymbol{r}+\Delta \phi_{1}\right) \hat{\boldsymbol{e}}_{x} .
\end{aligned}
$$

A intensidade luminosa $I_{1}\left(\mathrm{P}_{1}\right)$ em um ponto $\mathrm{P}_{1}$ qualquer do anteparo 1 é proporcional ao valor médio do módulo ao quadrado do campo elétrico resultante naquele ponto do anteparo:

$$
I_{1}\left(P_{1}\right) \propto<\left[\mathbf{E}_{1}\left(P_{1}, t\right)+\mathbf{E}_{2}\left(P_{1}, t\right)\right]^{2}>
$$

onde o símbolo $\langle\ldots\rangle$ corresponde uma média temporal realizada durante um período $(T)$ de oscilação dos campos elétricos; ou seja

$$
\begin{aligned}
& <\left[\mathbf{E}_{1}\left(P_{1}, t\right)+\mathbf{E}_{2}\left(P_{1}, t\right)\right]^{2}>=\frac{1}{T} \int_{0}^{T}\left[\mathbf{E}_{1}\left(P_{1}, t\right)+\mathbf{E}_{2}\left(P_{1}, t\right)\right] \cdot\left[\mathbf{E}_{1}\left(P_{1}, t\right)+\mathbf{E}_{2}\left(P_{1}, t\right)\right] d t= \\
& \frac{1}{T} \int_{0}^{T}\left[E_{1}^{2}\left(P_{1}, t\right)+2 \mathbf{E}_{1}\left(P_{1}, t\right) \cdot \mathbf{E}_{2}\left(P_{1}, t\right)+E_{2}^{2}\left(P_{1}, t\right)\right] d t= \\
& \frac{E_{0}^{2}}{2 T} \int_{0}^{T}\left[\cos ^{2}\left(\omega t-\boldsymbol{k}_{1} \cdot \boldsymbol{r}\right)+2 \cos \left(\omega t-\boldsymbol{k}_{1} \cdot \boldsymbol{r}\right) \cos \left(\omega t-\boldsymbol{k}_{1} \cdot \boldsymbol{r}+\Delta \phi_{1}\right)+\cos ^{2}\left(\omega t-\boldsymbol{k}_{1} \cdot \boldsymbol{r}+\Delta \phi_{1}\right)\right] d t
\end{aligned}
$$

É fácil mostrar que a primeira e a terceira das integrais do lado direito dessa expressão são iguais a $T / 2$. Para encontrar o resultado da segunda integral, usamos a identidade trigonométrica

$$
\cos A \cos B \equiv \frac{1}{2}[\cos (A-B)-\cos (A+B)]
$$

com a qual se demonstra que a segunda integral é igual a $T \cos \Delta \phi_{1}$. Com isso,

$$
\begin{aligned}
& <\left[\mathbf{E}_{1}\left(P_{1}, t\right)+\mathbf{E}_{2}\left(P_{1}, t\right)\right]^{2}>=E_{o}^{2}\left(1+\cos \Delta \phi_{1}\right) \equiv \\
& 2 E_{o}^{2} \cos ^{2}\left(\frac{\Delta \phi_{1}}{2}\right)
\end{aligned}
$$

de onde segue que

$$
I_{1}\left(P_{1}\right)=2 I_{0} \cos ^{2}\left(\frac{1}{2} a \rho^{2}\right),
$$

pois $I_{0} \propto E_{0}^{2} / 2$ [13]. Por um procedimento de cálculo muito parecido, mostra-se que a intensidade $I_{2}\left(\mathrm{P}_{2}\right) \mathrm{em}$ um ponto não central do anteparo 2 é dada por

$$
I_{2}\left(P_{2}\right)=2 I_{0} \operatorname{sen}^{2}\left(\frac{1}{2} a \rho^{2}\right) .
$$

As Eqs. (1) e (2) diferem por um fator $1 / 2$ das correspondentes expressões encontradas em livros textos [13] para o caso de fenda dupla. Isso se deve ao fato de que, nos livros, se está considerando a interferência entre duas fendas de intensidade $I_{0}$ cada uma; enquanto que aqui, para um ponto de um dos anteparos de um IMZ, o interferômetro equivale a um arranjo de fenda dupla em que cada fonte fictícia tem intensidade igual a $I_{0} / 2$ apenas.

Essas duas relações, portanto, são as equações do caso default. Elas significam que, em todos os pontos de um anteparo situados a uma mesma distância $\rho$ do centro do mesmo - e que constituem, portanto, um círculo - a intensidade é igual. Logo, as "franjas" de interferência construtiva (ou destrutiva) serão anéis claros (ou escuros) concêntricos ao centro do anteparo, cada qual com intensidade dada pela função $\cos ^{2}\left(\frac{1}{2} a \rho^{2}\right)$ e todos com a mesma intensidade máxima $2 I_{0}$.

\subsection{Introduzindo polaróides}

Consideremos agora o caso em que dois filtros polaróides $\mathrm{FP}_{1}$ e $\mathrm{FP}_{2}$ são introduzidos nos braços 1 e 2 de um IMZ, respectivamente, com seus eixos de polarização formando ângulos $\xi_{1}$ e $\xi_{2}$ com a direção horizontal da polarização da fonte. Após a luz ter se dividido no primeiro semi-espelho, percorrido os braços do IMZ e passado pelo segundo semi-espelho, chegando a um ponto $\mathrm{P}_{1}$ qualquer do anteparo 1 , o campo elétrico $\mathbf{E}_{1}$ da onda proveniente do braço 1 e o campo elétrico $\mathbf{E}_{2}$ da onda proveniente do braço 2 podem ser representados, respectivamente, pelos vetores

$$
\mathbf{E}_{1}\left(P_{1}, t\right)=\frac{E_{0}}{\sqrt{2}} \cos \xi_{1} \cos \left(\omega t-\boldsymbol{k}_{1} \cdot \boldsymbol{r}\right) \hat{\boldsymbol{e}}_{1}
$$

$\mathrm{e}$

$$
\mathbf{E}_{2}\left(P_{1}, t\right) \cong \frac{E_{0}}{\sqrt{2}} \cos \xi_{2} \cos \left(\omega t-\boldsymbol{k}_{1} \cdot \boldsymbol{r}+\Delta \phi_{1}\right) \hat{\boldsymbol{e}}_{2}
$$


onde $\hat{\boldsymbol{e}}_{1}$ e $\hat{\boldsymbol{e}}_{2}$ são os vetores unitários dos eixos de polarização de $\mathrm{FP}_{1}$ e de $\mathrm{FP}_{2}$, respectivamente. A situação default, de fato, é um caso particular dessa situação mais geral, quando $\xi_{1}=\xi_{2}=0$ (e considerando-se que os filtros polarizadores sejam ideais).

Com essa modificação, em um ponto $\mathrm{P}_{1}$ qualquer do anteparo 1, por exemplo, a intensidade fica dada por

$$
\begin{aligned}
& I_{1}\left(P_{1}\right)=<\left[\mathbf{E}_{1}\left(P_{1}, t\right)+\mathbf{E}_{2}\left(P_{1}, t\right)\right]^{2}>=\frac{1}{T} \int_{0}^{T}\left[E_{1}^{2}\left(P_{1}, t\right)+2 \mathbf{E}_{1}\left(P_{1}, t\right) \cdot \mathbf{E}_{2}\left(P_{1}, t\right)+E_{2}^{2}\left(P_{1}, t\right)\right] d t \\
& =\frac{E_{0}^{2}}{2 T} \int_{0}^{T}\left[\cos ^{2}\left(\xi_{1}\right) \cos ^{2}\left(\omega t-\boldsymbol{k}_{1} \cdot \boldsymbol{r}\right)+2 \cos \left(\xi_{1}\right) \cos \left(\xi_{2}\right) \cos \left(\omega t-\boldsymbol{k}_{1} \cdot \boldsymbol{r}\right) .\right. \\
& \left.\cos \left(\omega t-\boldsymbol{k}_{1} \cdot \boldsymbol{r}+\Delta \phi_{1}\right) \cos (\Delta \xi)+\cos ^{2}\left(\xi_{2}\right) \cos ^{2}\left(\omega t-\boldsymbol{k}_{1} \cdot \boldsymbol{r}+\Delta \phi_{1}\right)\right] d t,
\end{aligned}
$$

onde $\Delta \xi=\xi_{1}-\xi_{2}$ é o ângulo que os eixos de polarização de $\mathrm{FP}_{1}$ e $\mathrm{FP}_{2}$ formam entre si, de modo que $\cos (\Delta \xi)$ $=\hat{e}_{1} \cdot \hat{e}_{2}$. As integrações a ser feitas são basicamente as mesmas encontradas no caso default, mas os fatores multiplicativos independentes do tempo agora são diferentes. Efetuando as integrais, usando um pouco de álgebra e trigonometria básicas, pode-se mostrar que $I_{1}\left(P_{1}\right)$ é dada por

$$
I_{1}\left(P_{1}\right)=\frac{1}{2} I_{o}\left[\cos ^{2} \xi_{1}+\cos ^{2} \xi_{2}+2 \cos \xi_{1} \cos \xi_{2} \cos (\Delta \xi) \cos \left(a \rho^{2}\right)\right]
$$

Para obter $I_{2}\left(\mathrm{P}_{2}\right)$, basta efetuar a substituição $\Delta \phi_{1} \rightarrow \Delta \phi_{2}=\Delta \phi_{1}+\pi$ na expressão inicial de $I_{1}\left(\mathrm{P}_{1}\right)$. Entretanto, não precisamos efetuar novamente todas as contas, pois isso equivale à substituição $a r^{2} \rightarrow a r^{2}+\pi$ na expressão final de $I_{1}\left(\mathrm{P}_{1}\right)$ mostrada acima. Com isso, rapidamente se obtém

$$
\begin{gathered}
I_{2}\left(P_{2}\right)=\frac{1}{2} I_{o}\left[\cos ^{2} \xi_{1}+\cos ^{2} \xi_{2}-\right. \\
\left.2 \cos \xi_{1} \cos \xi_{2} \cos (\Delta \xi) \cos \left(a \rho^{2}\right)\right] .
\end{gathered}
$$

Note que, quando $\xi_{1}=\xi_{2}=\Delta \xi=0$, as expressões acima se reduzem às correspondentes expressões para o caso default. Além disso, com um pouco de álgebra pode-se demonstrar que as posições dos máximos e mínimos não mudam em relação às do caso default, de maneira que o padrão observado no anteparo não tem sua geometria alterada. A variação dos valores de $\xi_{1} \mathrm{e}$ $\xi_{2}$ e, conseqüentemente, de $\Delta \xi$, apenas diminui a intensidade dos anéis laterais concêntricos em relação à intensidade do máximo central, mas não altera a posição dos mesmos em relação às suas posições na situação default. Um caso de grande interesse é quando $\xi_{1}=0$, $\xi_{2}=\Delta \xi=90^{\circ}$, em que $I_{1}\left(\mathrm{P}_{1}\right)$ e $I_{2}\left(P_{2}\right) \rightarrow \frac{1}{2} I_{o}=$ constante. Nenhum padrão de interferência seria observado nos anteparos, neste caso, apenas uma iluminação uniforme ao longo dos mesmos. Ou seja, dois feixes luminosos idênticos em tudo, mas com polarizações perpendiculares entre si, não interferem, o que está em concordância com o que se observa na natureza.

Outro caso de interesse é quando um terceiro filtro polaróide, $\mathrm{FP}_{3}$, é introduzido na saída do IMZ, ou seja, entre o segundo semi-espelho e o anteparo 1. Seja $\xi_{3}$ o ângulo formado pelo eixo de polarização do novo polaróide com o eixo $x$, e $\hat{e}_{3}$ o vetor unitário da direção do eixo de polarização de $\mathrm{FP}_{3}$. Os campos elétricos das ondas eletromagnéticas que se combinam em um ponto $\mathrm{P}_{1}$ qualquer do anteparo 1 , depois de seguirem pelos braços 1 e 2, são dados, respectivamente, pelas expressões

$$
\mathbf{E}_{1}\left(P_{1}, t\right)=\frac{E_{0}}{\sqrt{2}} \cos \xi_{1} \cos \xi_{3} \operatorname{sen}\left(\omega t-\boldsymbol{k}_{1} \cdot \boldsymbol{r}\right) \hat{\boldsymbol{e}}_{3}
$$

e

$$
\mathbf{E}_{2}\left(P_{1}, t\right) \cong \frac{E_{0}}{\sqrt{2}} \cos \xi_{2} \cos \xi_{3} \operatorname{sen}\left(\omega t-\boldsymbol{k}_{1} \cdot \boldsymbol{r}+\Delta \phi_{1}\right) \hat{\boldsymbol{e}}_{3} .
$$

Por um procedimento de cálculo bastante parecido com o que efetuamos antes, pode-se demonstrar que

$$
\begin{array}{r}
I_{1}\left(P_{1}\right)=\frac{1}{2} I_{0}\left\{\cos ^{2} \xi_{1} \cos ^{2} \xi_{13}+\cos ^{2} \xi_{2} \cos ^{2} \xi_{23}+\right. \\
\left.2 \cos \xi_{1} \cos \xi_{2} \cos \xi_{13} \cos \xi_{23} \cos \left(a r^{2}\right)\right\}
\end{array}
$$

onde $\xi_{13}=\left|\xi_{3}-\xi_{1}\right|$ e $\xi_{23}=\left|\xi_{3}-\xi_{2}\right|$. É fácil verificar que este caso se reduz ao caso default no limite $\xi_{1}, \xi_{2}$ e $\xi_{3} \rightarrow 0$, mas $n \tilde{a} o$ se reduz ao caso anterior (dos dois filtros polaróides colocados um em cada braço) quando apenas $\xi_{3}$ tende a zero, o que justifica o termos tratado como um caso independente do anterior.

\subsection{Retirando o segundo semi-espelho}

A ocorrência de interferência entre os feixes luminosos provenientes dos braços 1 e 2 depende fundamentalmente da presença do segundo semi-espelho, que atua com uma espécie de "recombinador" dos feixes nos dois anteparos. Se ele não estiver presente, apenas o feixe 
proveniente do braço 1 chegará ao anteparo 1, e apenas o feixe do braço 2 chegará ao anteparo 2. Portanto, na ausência de polaróides, $I_{1}\left(\mathrm{P}_{1}\right)=I_{2}\left(\mathrm{P}_{2}\right)=I_{o}$, o que corresponde a uma iluminação uniforme sobre os anteparos. Para o caso em que os polaróides $\mathrm{FP}_{1}$ e $\mathrm{FP}_{2}$ estão presentes, de acordo com a lei de Malus, teremos,

$$
I_{1}\left(P_{1}\right)=I_{o} \cos ^{2}\left(\xi_{1}\right) \quad \text { e } \quad I_{2}\left(P_{2}\right)=I_{o} \cos ^{2}\left(\xi_{2}\right)
$$

\subsection{Efeitos de difração}

Uma fonte luminosa real não é realmente puntiforme. Vamos considerar agora que ela seja uma fenda circular de raio $R$, por onde sai um feixe de laser polarizado horizontalmente. Sendo uma fonte extensa, ocorrerá interferência mesmo entre raios luminosos emitidos por diferentes pontos de uma mesma abertura circular, o que dará origem a efeitos de difração nos padrões observados nos anteparos [13]. Tais efeitos podem ser incorporados facilmente ao modelo anterior, bastando que, em todas as expressões encontradas antes, se substitua o termo constante $I_{0}$ pela função de Airy [22],

$$
I_{0} \rightarrow I_{\text {Airy }} \equiv\left(\frac{2 J_{1}(x)}{x}\right)^{2}
$$

onde $J_{1}(x)$ é a função de Bessel de primeira classe e de ordem 1 [22], com argumento dado por

$$
x=\frac{2 \pi R}{\lambda} \operatorname{sen} \theta .
$$

Dessa maneira, a função de Airy nos fornece a intensidade local do feixe na entrada de cada braço do IMZ (quando não ocorreu ainda a interferência entre os feixes, mas tão somente difração na abertura da fonte) ou, alternativamente, a intensidade em um ponto qualquer dos anteparos quando nenhum filtro polaróide foi introduzido e quando o segundo semi-espelho não se encontra presente, não existindo interferência nos anteparos e nenhuma perda de energia por absorção em polaróides. Novamente, como $\theta$ é um ângulo pequeno, podemos aproximá-lo pelo valor de sua tangente,

$$
\operatorname{tg} \theta=\frac{\rho}{2 L+D}
$$

de modo que $x \cong b \rho$, onde $b=\frac{2 \pi R}{\lambda(2 L+D)}$ é uma constante.

Com isso, para o caso default, por exemplo, as intensidades em um ponto $\mathrm{P}_{1}$ do anteparo 1 e em um ponto $\mathrm{P}_{2}$ do anteparo 2 ficam dadas, respectivamente, pelas expressões

$$
I_{1}\left(P_{1}\right)=2 I_{\text {Airy }}(b \rho) \cos ^{2}\left(\frac{1}{2} a \rho^{2}\right)
$$

e

$$
I_{2}\left(P_{2}\right)=2 I_{\text {Airy }}(b \rho) \operatorname{sen}^{2}\left(\frac{1}{2} a \rho^{2}\right)
$$

A Fig. 3 mostra os gráficos de $I_{1}\left(\mathrm{P}_{1}\right)$ para os casos default correspondentes a uma fonte puntiforme e outra extensa, superpostos. Os máximos de interferência produzidos por duas fendas circulares - máximos da função $\cos ^{2}\left(\frac{1}{2} a \rho^{2}\right)$-, todos de mesma intensidade e em forma de anéis concêntricos, tornam-se modulados pela função de Airy, a qual também possui máximos laterais correspondentes a anéis, mas com intensidades cada vez menores. Dessa maneira, o padrão resultante é formado por um disco brilhante - o máximo central de interferência que fica no centro do máximo central da função de Airy e é modulado por ela - cercado de anéis de interferência situados dentro de anéis do padrão de difração de Airy. Observe que o valor de pico do disco central de Airy é muito maior do que os valores de pico laterais do padrão de difração. Isso significa que, na prática, apenas os anéis de interferência que cabem dentro do disco central de difração são facilmente visíveis, com os próximos anéis de interferência tão fracos, devido à atenuação produzida pela função de Airy, que praticamente não são notados no anteparo.
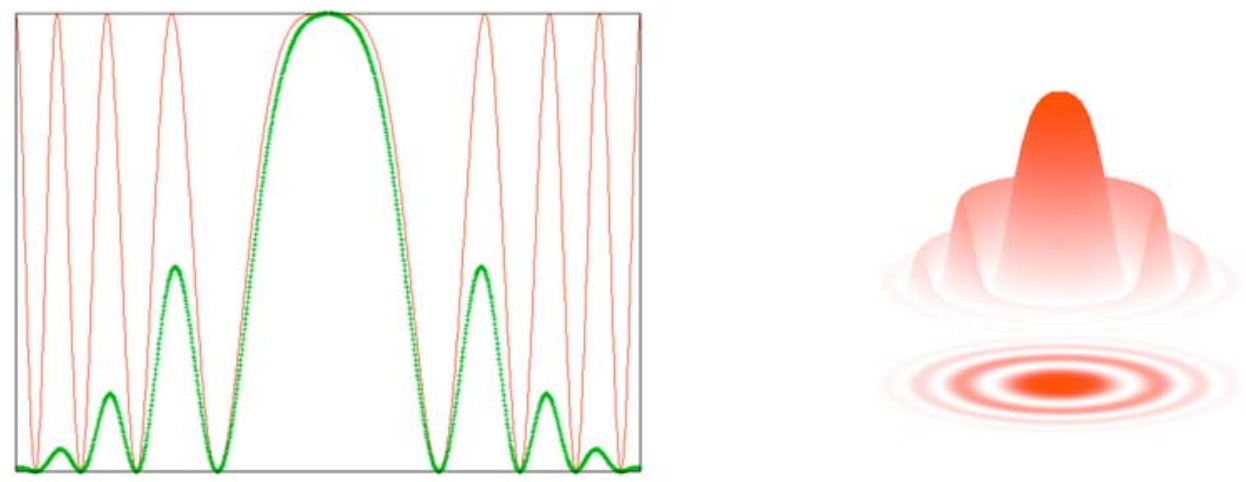

Figura 3 - Lado esquerdo: Gráfico da intensidade $I_{1}\left(\mathrm{P}_{1}\right)$ para os casos de fonte puntiforme (todos os anéis de interferência com a mesma intensidade) e de fonte extensa (anéis progressivamente mais fracos, sendo o quarto deles tão fraco que praticamente não é notado). O máximo central e os três primeiros máximos laterais de interferência caem dentro do máximo central de difração. Lado direito: figura tridimensional correspondente ao gráfico do lado esquerdo. O padrão de anéis observado no anteparo também é mostrado para comparação. 
A Fig. 4 mostra a aparência na tela de um computador de um padrão originado no anteparo 1. Tal padrão corresponde à situação em que os valores dos parâmetros $L, D, \lambda$ e $R$ foram escolhidos tais que exatamente 9 máximos de interferência (um central e oito laterais) de fenda dupla cabem dentro do máximo central da função de Airy, com o quinto máximo lateral de interferência coincidindo exatamente com o primeiro mínimo da função de Airy.

Para o caso em que um polaróide é introduzido em cada braço do IMZ (e a fonte de laser não é puntiforme), $I_{1}\left(\mathrm{P}_{1}\right)$ e $I_{2}\left(\mathrm{P}_{2}\right)$ ficam dadas pelas expressões

$$
\begin{aligned}
& I_{1}\left(P_{1}\right)=\frac{1}{2} I_{\text {Airy }}(b \rho)\left[\cos ^{2} \xi_{1}+\cos ^{2} \xi_{2}+\right. \\
& \left.2 \cos \xi_{1} \cos \xi_{2} \cos (\Delta \xi) \cos \left(a \rho^{2}\right)\right]
\end{aligned}
$$

e

$$
\begin{aligned}
& I_{2}\left(P_{2}\right)=\frac{1}{2} I_{\text {Airy }}(b \rho)\left[\cos ^{2} \xi_{1}+\cos ^{2} \xi_{2}-\right. \\
& \left.2 \cos \xi_{1} \cos \xi_{2} \cos (\Delta \xi) \cos \left(a \rho^{2}\right)\right]
\end{aligned}
$$

Também é fácil mostrar que essas funções se reduzem à função de Airy quando $\Delta \xi$ tende a $90^{\circ}$ (ou $\xi_{2}=\xi_{1}$ $\left.+90^{\circ}\right)$, resultando em uma iluminação não uniforme nos anteparos, mas sem franjas, correspondente ao disco central de Airy (os anéis laterais dessa função são muito fracos para serem notados nos anteparos; veja o gráfico da função de Airy).

O cálculo mostrado nessa seção foi usado para simular um IMZ em regime clássico. Com a devida interpretação de amplitudes de probabilidades de Max Born [14, 15], esse cálculo também serviu de base para a simulação do IMZ no regime em que um único fóton incide no IMZ de cada vez, ou seja, o regime monofotônico.

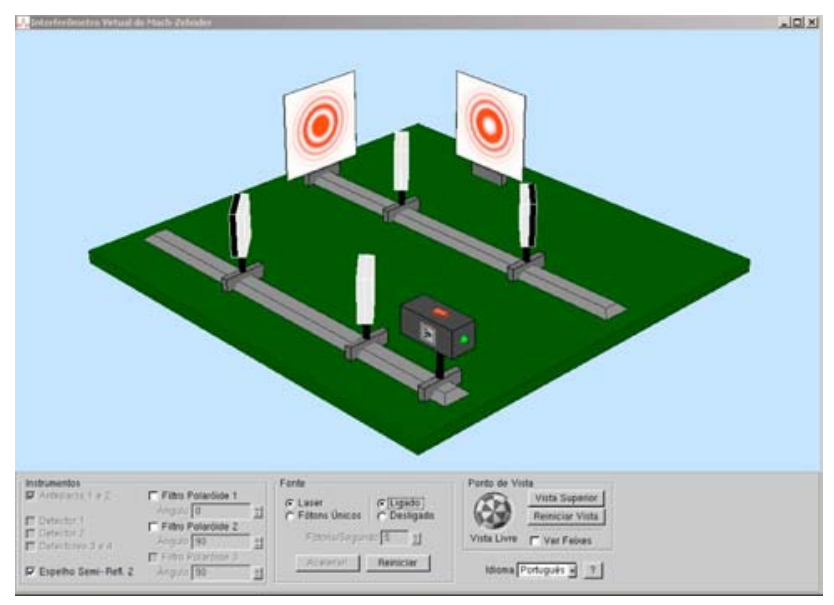

Figura 4 - Tela da situação default para o software do IMZ operando em regime clássico.

\section{Um panorama do IMZ virtual em re- gime clássico}

O Interferômetro Virtual de Mach-Zehnder é um software educacional de $372 \mathrm{~KB}$ disponível na rede [19] para o sistema Windows e de fácil utilização. O aplicativo pode ser usado em três idiomas, a saber, português, espanhol e inglês. Ele é uma versão mais aprimorada da simulação de um IMZ, além de modernizada em recursos visuais, do que a de um software produzido por Müller e Wiesner [6,7]. Em linhas gerais, o software simula o IMZ no regime da óptica ondulatória (tela mostrada na Fig. 4) e no regime monofotônico, ou seja, em pleno regime quântico. Comparado ao software pioneiro de Müller e Wiesner, ressaltamos de imediato que não há uma correspondência física perfeita entre os dois casos, uma vez que, em nosso trabalho, o feixe de laser é considerado polarizado (o que é mais realístico), enquanto no caso simulado pelos autores citados o feixe de laser é considerado como não polarizado. Nosso simulador permite analisar com os polaróides uma série de situações mais ricas e diversificadas em relação ao software de Wiesner e Müller. Outra diferença fundamental no nosso software foi permitir ao usuário a opção de retirada do segundo semi-espelho, combinada ao uso dos polaróides. Essa opção permite a identificação da direção de polarização do feixe, ao mesmo tempo em que ressalta a importância do segundo semiespelho para a formação do padrão de interferência. Esse recurso de retirada do segundo semi-espelho tem sido explorado na literatura no que se refere principalmente à discussão da dualidade onda-partícula, em regime quântico [4, 5, 8-10]. Nesta seção, mostraremos alguns recursos do software, operando em regime clássico, que enfatizam os conceitos acerca da natureza ondulatória da luz e as condições necessárias para a observação do fenômeno de interferência. Ressaltamos, no entanto, que há inúmeras outras situações a serem exploradas pelo estudante ou pelo professor, de maneira que, não esgotamos aqui, de modo algum, todo o potencial do software, uma vez que não é este também nosso objetivo. A idéia de um simulador do tipo bancada com múltiplas opções é que o aluno ou professor possa usar também sua própria curiosidade e sua criatividade para propor diferentes arranjos de espelhos e polaróides que lhe permitam experimentar o caráter ondulatório da luz sob diferentes simulações. Neste sentido, um roteiro exploratório bastante detalhado, já testado em sala de aula, em cursos de introdução à MQ do Mestrado Profissional em Ensino de Física da UFRGS, pode ser encontrado na Ref. [23].

Deslizando o mouse sobre a bancada virtual, todos os espelhos, semi-espelhos e anteparos podem ser identificados. O usuário pode também escolher o ângulo de perspectiva que lhe seja mais conveniente para trabalhar, assim como poderá ver o caminho seguido pelos feixes com a opção "ver feixes". Ao selecionar a fonte 
"Laser" e depois acionar o botão "Ligado", o IMZ passa a funcionar em regime clássico, exibindo automaticamente uma tela como a da Fig. 4. Os anteparos 1 e 2 revelam padrões de interferência complementares, estando iluminada a região central do anteparo 1 (interferência construtiva), e não-iluminada a correspondente região do anteparo 2 (interferência destrutiva), conforme se pode esperar pelos padrões descritos pelas Eqs. (5). Observe que, na situação default, o segundo semi-espelho é mantido.

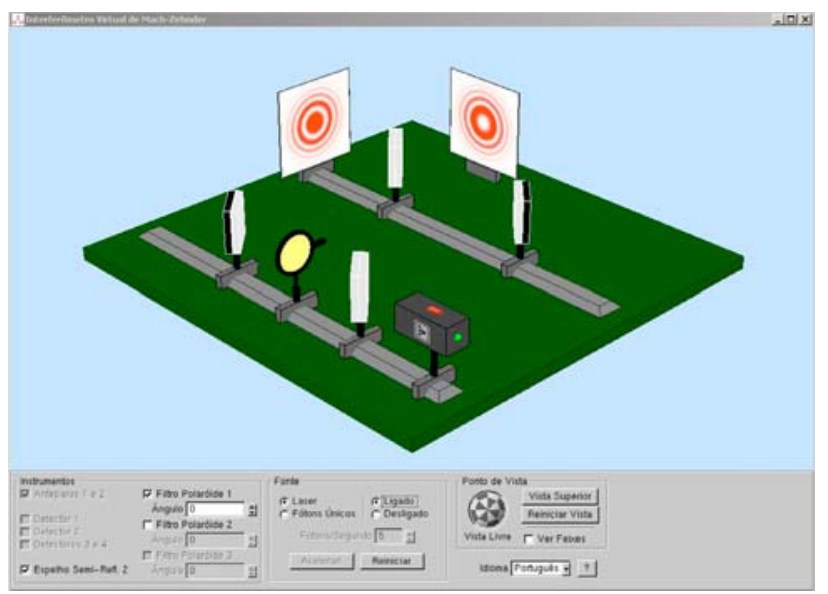

Figura 5 - O IMZ exibindo anéis de interferência quando apenas um polaróide está presente, com seu eixo de polarização paralelo à direção de polarização do feixe.

O software oferece também a possibilidade de utilização, simultânea ou não, de um filtro polaróide em cada braço. Isso permite ao usuário a visualização de um padrão de anéis que pode ser reforçado, enfraquecido ou mesmo destruído, à medida que ele faz variar o ângulo relativo entre os polaróides, embora as posições dos anéis de interferência não sejam alteradas.

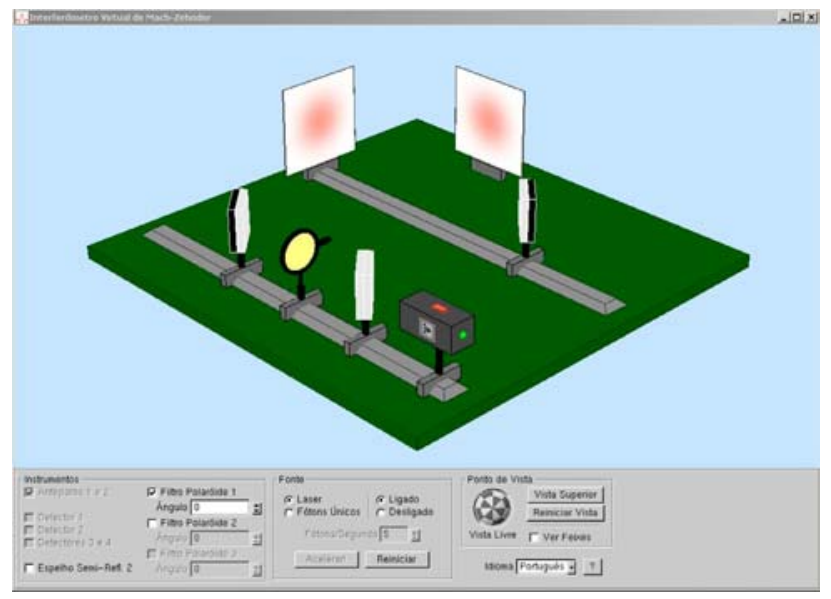

Figura 6 - Destruição dos anéis de interferência com a retirada do segundo semi-espelho.

Compare agora, o resultado do arranjo da Fig. 6 (sem o segundo semi-espelho) com o da Fig. 5 (com o segundo semi-espelho presente). A retirada do segundo semi-espelho faz com que o feixe, embora ainda sendo dividido no primeiro semi-espelho, e ainda rumando através dos dois braços, não seja mais colocado em superposição sobre os anteparos, o que acarreta o desaparecimento do padrão de interferência. Reiteramos que o polaróide 1 não altera o resultado dessa experiência, pois o feixe é totalmente transmitido pelo polaróide, conforme constatamos na Fig. 5. O polaróide permanece nessa simulação somente para a comparação entre a situação da Fig. 5 e da Fig. 6. O arranjo da Fig. 6 é, no entanto, totalmente análogo à situação do IMZ quando o polaróide 1 não está presente e o segundo semi-espelho é removido.

Outro caso bastante ilustrativo é o caso em que os eixos de polarização de $\mathrm{FP}_{1}$ e de $\mathrm{FP}_{2}$ estão "cruzados", ou seja, formando $90^{\circ}$ entre si. Os padrões de interferência nos anteparos desaparecem automaticamente, de acordo com a Lei de Malus [13,14], quando o eixo de polarização do polaróide de um dos braços é girado em $90^{\circ}$ com respeito ao eixo do polaróide do outro braço. O padrão de intensidade luminosa em qualquer dos anteparos, portanto, deve ser o mesmo da Fig. 6, mas com a intensidade reduzida à metade porque o feixe que entra no braço correspondente do interferômetro acaba sendo inteiramente absorvido pelo seu polaróide, o que reduz à metade a intensidade luminosa do padrão em relação à do padrão mostrado na Fig. 6. Ou seja, para o caso de polaróides cruzados, devemos esperar que $I_{1}\left(\mathrm{P}_{1}\right)=I_{2}\left(\mathrm{P}_{2}\right)=\frac{1}{2} I_{\text {Airy }}(b \rho)$, o que, de fato, resulta das relações (7) para o caso em que $\xi_{2}=\xi_{1}+$ $90^{\circ}$.

A opção de retirada do segundo semi-espelho aliada ao uso de polaróides cruzados gera um conjunto de novas possibilidades exploratórias, tanto por parte do professor quanto do estudante.

\section{Considerações finais}

Neste trabalho apresentamos, com detalhes, o tratamento matemático de um IMZ em regime clássico. Tal discussão, embora análoga à da fenda dupla, é pouco explorada na literatura, no Brasil ou no exterior. No entanto, esse arranjo experimental vem merecendo crescente interesse, tanto daqueles que trabalham em áreas tecnológicas quanto dos físicos teóricos que desenvolvem pesquisas sobre os fundamentos da FQ, por representar uma releitura mais moderna do experimento de Young. Do ponto de vista do ensino de física, também nos últimos anos sua utilização vem recebendo maior espaço. Em nosso caso, isso resultou no desenvolvimento de um software educacional que simula o funcionamento de um IMZ, o que exigiu que desenvolvêssemos o tratamento matemático do regime clássico, apresentado na seção 2, para obtenção dos resultados físicos corretos. Um dos objetivos desse simulador é, partindo da óptica ondulatória, fazer uma ponte para discussões do IMZ operando em regime quântico. Nesse sentido, tal aparato parece ser mais interessante do que o da 
fenda dupla de Young, pois permite uma reflexão sobre o fenômeno da interferência quântica a partir do problema conceitual acerca da escolha de caminhos pelo fóton. Naturalmente, entender esse regime quântico requer a compreensão dos resultados do paradigma ondulatório contido nesse experimento, o que justifica o tratamento clássico aqui apresentado em detalhes.

\section{Agradecimentos}

Fernanda Ostermann agradece ao CNPq pelo apoio parcial e pelos recursos obtidos no Edital Universal 08/2004. Os autores agradecem a Leandro Augusto Frata Fernandes pelo excelente trabalho de informática na confecção do software.

\section{Referências}

[1] R. Cleve, A. Ekert, L. Henderson, C. Macchiavello and M. Mosca, Complexity 4, 33 (1998).

[2] G.E.M. Cabral, A.F. de Lima, B. Lula Jr., Rev. Bras. Ens. Fis. 26, 109 (2004).

[3] K.P. Zetie, S.F. Adams and R.M. Tocknell, Physics Education 35, 46 (2000).

[4] O. Pessoa Jr., Rev. Bras. Ens. Fis. 19, 27 (1997).

[5] O. Pessoa Jr., Conceitos de Física Quântica (Livraria da Física, São Paulo, 2003).

[6] R. Müller and H. Wiesner, Am. J. Phys. 70, 200 (2002).

[7] R. Müller and H. Wiesner, Interferômetro de MachZehnder, simulador disponível em http://www. physik.uni-muenchen.de/didaktik/Computer/interfer/ interfere.html.

[8] T.F. Ricci e F. Ostermann, Uma Introdução Conceitual à Mecânica Quântica para Professores do Ensino Médio (Instituto de Física da UFRGS, Porto Alegre, 2003), Textos de Apoio ao professor de Física, n. 14.
[9] F. Ostermann e T.S.F. Ricci, Atas do IV Encontro Nacional de Pesquisa em Educação em Ciências (Bauru, SP, 2003), em CD-ROM.

[10] F. Ostermann e S.D. Prado, Rev. Bras. Ens. Fis. 27, 193 (2005)

[11] L. Zehnder, Z. Instrumentenkd. 11, 275 (1891).

[12] L. Mach, Z. Instrumentenkd 12, 89 (1892).

[13] D. Halliday, R. Resnick and J. Walker, Fundamentals of Physics Extended (John Wiley \& Sons, 1997), $5^{a}$ ed.

[14] P.A. Tipler, Física Moderna (Editora Livros Técnicos e Científicos, Rio de Janeiro, 2001), Física - Óptica e Física Moderna (Editora Guanabara Koogan, Rio de Janeiro, RJ), v. 4, $3^{a}$ ed.

[15] R. Eisberg e R. Resnick, Física Quântica (Editora Campus, São Paulo, 1994).

[16] R.P. Feynman, R.B. Leighton and M. Sands, The Feynman Lecture on Physics (Addison-Wesley, Reading, 1963) v. 3, cap. 37.

[17] Peter Rodgers, Editorial de Physics World, p. 15, setembro 2002. Disponível em http://physicsweb.org/ articles/world/15/9/1/1.

[18] http://www.stanford.edu/group/chugroup/amo/inter ferometry.html.

[19] F. Ostermann et al., Interferômetro Virtual de Mach-Zehnder, simulador disponível em http://www. if.ufrgs.br/ fernanda/IMZ.

[20] F. Ostermann, S.D. Prado e T.S.F. Ricci, Física na Escola 7(1), 22 (2006).

[21] V. Degiorgio, Am. J. Phys. 48, 81 (1980).

[22] M. Abramowitz and I.A. Stegun, Airy Functions §10.4, in Handbook of Mathematical Functions with Formulas, Graphs, and Mathematical Tables (Dover, Nova York, 1972), p. 446-452.

[23] F. Ostermann e T.S.F. Ricci, Caderno Brasileiro de Ensino de Física 22, 9 (2005). 